Recepción: 13 / 08 / 2017

Aceptación: 09 / 10 / 2017

Publicación: 15 / 12 / 2017

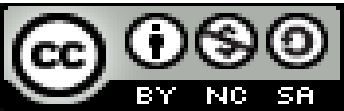

Ciencias sociales y políticas

Artículo de investigación

\title{
Plan de transformación del borde costero para el desarrollo del cantón de Jaramijó
}

Plan of transformation of the coastal edge for the development of the canton of Jaramijó

Plano de transformação da orla costeira para o desenvolvimento do cantão de Jaramijó

\author{
Marcos R. Gallo-Zambrano ${ }^{\mathrm{I}}$ \\ arqmarcosg@hotmail.com \\ Juan R. Cedeño-Candela II \\ jcandela_24@hotmail.com
}

Correspondencia: arqmarcosg@hotmail.com

\begin{abstract}
IMagister en Gestión Ambiental, Magister en Arquitectura Mención Diseño Urbano, Arquitecto, Docente de la Universidad Laica Eloy Alfaro de Manabí, Manta, Ecuador.

II Magister en Gestión Ambiental, Arquitecto, Docente de la Universidad Laica Eloy Alfaro de Manabí, Manta, Ecuador.
\end{abstract}




\title{
Resumen
}

La falta de una planificación urbana, en el cantón de Jaramijó, es notable ya que carece de toda necesidad social, espacial, ambiental, cultural y recreacional. A tal efecto, el presente artículo propone un plan de transformación del borde costero para el desarrollo de la ciudad de Jaramijó. La investigación fue de tipo descriptivo y documental. Bajo una modalidad de campo, dado que la información se obtuvo a través de un estudio sistemático de los hechos en el mismo borde costero del cantón Jaramijó, para ampliar, conocer y profundizar la información se utilizaron libros, revistas y periódicos como medio bibliográfico de información y datos que permitieron generar los resultados expuestos, todos estos instrumentos de generación de información constituyeron el corpus del estudio. La técnica para la interpretación y presentación de la información fue la hermenéutica. Los resultados evidenciaron la falta de estudios que consideren los problemas urbanísticos y políticos del borde costero del cantón Jaramijó, por lo que se presenta un plan de transformación. Entre sus conclusiones se pudo evidenciar la inexistencia de un ordenamiento de esta zona de investigación, debido a su gran extensión y alta densidad de población esparcida por todas partes, lo cual no ha permitido desarrollar las obras de infraestructuras básicas, los mobiliarios, los equipamientos, la identidad cultural y la falta de un desarrollo urbanístico que le permita consolidarse como una ciudad próspera al desarrollo turístico del cantón.

Palabras Clave: espacial; ambiental; cultural; recreacional; social; borde costero y servicios.

\begin{abstract}
The lack of urban planning, in the canton of Jaramijó, is remarkable since it lacks any social, spatial, environmental, cultural and recreational need. To this end, this article proposes a plan for the transformation of the coastal edge for the development of the city of Jaramijo. The research was descriptive and documentary. Under a field modality, given that the information was obtained through a systematic study of the facts in the same coastal edge of the Jaramijo county, in order to expand, know and deepen the information, books, magazines and newspapers were used as a bibliographic medium of information and data that allowed to generate the exposed results, all these instruments of generation of information constituted the corpus of the study. The technique for the interpretation and presentation of information was hermeneutics. The results
\end{abstract}



evidenced the lack of studies that consider the urban and political problems of the coastal edge of Jaramijo canton, for which a transformation plan is presented. Among its conclusions it was possible to demonstrate the lack of an ordering of this research area, due to its great extension and high density of population scattered everywhere, which has not allowed to develop the works of basic infrastructures, furniture, equipment, the cultural identity and the lack of an urban development that allows it to consolidate itself as a prosperous city to the tourist development of the canton.

Keywords: spatial; environmental; cultural; recreational; social; coastal and services.

\section{Resumo}

A falta de planejamento urbano, no cantão de Jaramijó, é notável, pois carece de qualquer necessidade social, espacial, ambiental, cultural e recreativa. Para tanto, este artigo propõe um plano para a transformação da orla costeira para o desenvolvimento da cidade de Jaramijo. A pesquisa foi descritiva e documental. Sob um campo de formulário, uma vez que a informação foi obtida através de um estudo sistemático dos fatos na mesma orla costeira do cantão Jaramijo a se expandir, conhecer e aprofundar as informações livros, revistas e jornais foram usados como meio de informação bibliográfica e dados que permitiram gerar os resultados expostos, todos esses instrumentos de geração de informações constituíram o corpus do estudo. A técnica para interpretação e apresentação de informações foi hermenêutica. Os resultados evidenciaram a falta de estudos que considerem os problemas urbanos e políticos da orla costeira do cantão Jaramijo, para os quais é apresentado um plano de transformação. Entre as suas conclusões foi evidente a ausência de um sistema desta área de pesquisa por causa de seu grande tamanho e alta densidade populacional espalhado por toda parte, o que não nos permitiu desenvolver as obras de infraestrutura básica, mobiliário, equipamentos, a identidade cultural e a falta de um desenvolvimento urbano que permita consolidar-se como uma cidade próspera para o desenvolvimento turístico do cantão.

Palavras chave: espacial; ambiental; cultural; recreacional; social; costeiro e serviços. 


\section{Introducción}

Jaramijó, alcanzo recientemente su cantonización (28 de abril de 1.998), se considera como el cantón más joven de la provincia de Manabí, antes parroquia del cantón Montecristi. La deficiencia urbana es notable, carece de toda necesidad social-espacial, ambiental recreacional y cultural. Aunque su desarrollo poblacional se basa en la producción pesquera artesanal, e industrial y una pequeña afluencia turística, que les permiten vivir de sus propios recursos. Sin embargo, hoy en día gran parte de la población vive en condiciones deprimentes; su economía, y el lenguaje del sentido urbanístico, ha impactado a los sectores más pobres de la ciudad. Posición, que actualmente siendo considerada como una las ciudades del Ecuador, debe de estar lista para afrontar los cambios que genera un estrato social y política que implica entrar en una nueva etapa y es allí donde la conducirá a la modernidad. Cabe destacar, que es notorio que la ciudad ha desarrollado un modelo de crecimiento y segregaciones sociales donde se demuestra que el cantón comenzó a sufrir transformaciones fundamentalmente en términos poblacionales, de crecimiento físico, económico y de la expansión de nuevas demandas y necesidades colectivas.

Esto último, se ha convertido en un gran problema para su población, al no recibir respuesta por parte de sus organismos gubernamentales, de quienes esperan que proporcione servicios que permita a la ciudad ser vivible, donde se tenga que hablar de un lenguaje en sentido urbanístico, que genere tenencias urbanísticas y para esto se necesita analizar su trazado urbano, cascos urbanos, etapas de crecimiento, morfología de las manzanas, morfología del espacios urbanos, áreas de crecimiento por extensión, áreas de polígonos vacíos, urbanismo, reordenamiento urbano, planificación, sistema urbano, asentamiento urbano, infraestructura urbana, estructura urbana, tipología de vivienda, vialidad urbana, suelo urbano, servicios públicos y equipamientos urbanos. Estas serán las necesidades, prioritarias a futuro y estarán marcadas en el contexto de la participación comunitaria, con nuevas propuestas que serán aplicados en el diario convivir, abriéndose a novedosas posibilidades que vayan enmarcados hacia un rumbo proporcional que establezca parámetros y leyes que tengan actitudes en desarrollo y de planificación para convertirla en una ciudad moderna para todos sus habitantes. La propuesta que se recomienda es que; se incorpore infraestructuras de servicios básicos, con el propósito de mejorar la calidad de 
vida de los moradores del sector permitiendo tener un desarrollo sustentable en beneficio del cantón y la provincia.

Es de destacar, que según datos históricos el crecimiento urbano que se registra en la ciudad de Jaramijó es muy grande: Como parroquia del cantón de Montecristi en el año de 1.968 contaba con 26,50 hectáreas; ubicada en la zona cercana a la playa donde su mayor actividad es la pesca artesanal. En 1.990 crece el tamaño a 46,80 hectáreas; en el 2.000 a 62,60 hectáreas, y en los actuales momentos cuenta con 97 hectáreas. La realidad de la ciudad de Jaramijó reside fundamentalmente en el hecho de que, por su reciente creación como cantón de la provincia de Manabí, no ha logrado superar sus deficiencias y problemas que prevalecen en el orden social, económico, cultural, ambiental, recreacional, vial, servicios básicos, por lo que es imprescindible emprender procesos con alternativas válidas que contribuyan a superar la patología a la que se hace alusión. (Órgano de difusión del gobierno Municipal del Cantón de Jaramijó, 2015).

Se hace necesario plantear un estudio que tenga sustentabilidad y un proceso evolutivo que permita racionalizar y ordenar el uso del suelo del borde costero mediante planes de equipamientos, mobiliarios, servicios básicos, espacio urbano, vial, recreacional, servicios públicos, saneamiento ambiental y turístico, logrando así el mejoramiento de la calidad de vida de sus habitantes y considerar muy bien la problemática y fundamentar las soluciones en base a una investigación que abarque todos los campos posible sin que se llegue a una confusión.

Cabe destacar, que entre los problemas que inciden en el desarrollo de la ciudad como cantón que apunta a crecer rápidamente, se pueden citar:

- $\quad$ Falta de política, planificación y tratamiento del borde costero.

- $\quad$ Falta de zonificación que acoja a las diferentes actividades del sector.

- $\quad$ Escasos niveles de equipamiento, mobiliarios, turismo y recreación.

- Deterioro del espacio natural.

- La falta de concienciación ciudadana.

- $\quad$ Pérdida de identidad histórica y cultural.

- Inseguridad ciudadana.

- Insalubridad. 
La solución al problema planteado, viene a partir de una eficaz gestión de los elementos que la estructuran tales como: estabilidad y provisión de recursos económicos, a lo que deberá sumar la adopción de estrategias en el proceso urbano y su ordenamiento sobre los requerimientos y las necesidades de los asentamientos humanos. Estos problemas en la actualidad están incidiendo directamente en el desarrollo social, económico, urbanístico, turístico y ambiental del cantón Jaramijo, toda vez que se constituye en grandes barreras que impiden el crecimiento y desarrollo urbano que exige el mundo moderno. Todo esto llevó a plantear una alternativa constructiva que contribuya a la recuperación del espacio urbano público, privado, turístico, ambiental, recreacional y cultural, como lo propone el plan de transformación del borde costero de Jaramijó, que se presenta en este artículo.

\section{Desarrollo}

Las operaciones transformadoras al ser reestructuradas no solo en sus aspectos formales y ambientales, sino en su funcionamiento y organización de movimiento, producen cambios en la red vial, donde se reestructura la circulación. A todo esto, se aprovecha para tomar la vía de circulación y darle nuevos aspectos con jardinerías, áreas peatonales, donde se mezclan varias actividades. Los nuevos espacios lineales como paseos, avenidas, autovías urbanas y grandes calles, dan un proceso de reorganización a todas las funciones circulatorias como los aspectos sociales y ambientales.

En muchos casos tienen aspectos positivos, en otros solo se considera lo circulatorio, con simples tratamientos en los bordes de los edificios, o se hacen recuperaciones forzadas, históricas, teniendo diferentes necesidades. La calle tradicional en la historia urbanística ha revelado como irrenunciable para la ciudad, es muy atractiva la recuperación de la calle peatonal como opción de uso limitado. Si se tratara de recuperar la calle tradicional, se iría directamente a la arquitectura y a la importancia de la forma construida que define la calle, esto demuestra que no hay ciudad sin calles, como tampoco calles sin arquitectura, recuperando la infinidad de formas en sus edificios marcando una continuidad en espacios longitudinal tridimensional. Todas estas características darían la pauta para una recuperación porque serían sendas, caminos, paseo o carretera, la calle adquiere su valor ambiental, formal, visualmente enriquecida y es capaz de agrupar varias funciones, sin renunciar a la circulación, convirtiéndose en un gran escenario. 
El Paisaje Urbano. Una ciudad se pude contemplar a lo lejos, desde una llanura, una montaña, o un avión. A veces el punto de vista está más abajo que el horizonte, en otros casos la vista es caballera. Cada ciudad, apunta Romero (2010), es diferente según su tamaño, función o edad. El interior de la ciudad tiene sus distintos paisajes. Según la zona de la ciudad, el centro, los barrios burgueses, industriales, los suburbios, y la periferia así será su aspecto. Cada una de las partes tiene su personalidad, condicionada por lo económico y lo social.

El lujo y la miseria, el ocio y el trabajo marcan el clima, determinan el medio ambiente de los espacios urbanos. Hay barrios vistosos, grises, alegres, tristes, amenos, y monótonos, que inspiran confianza. Otros en cambio resultan misteriosos y sombríos, producen miedo incluso pánico, sus paisajes son al fin de cuentas estados del alma colectiva. También hay ciudades o barrios en las grandes ciudades que permiten ver a lo lejos la naturaleza. Los puertos de mar o las ciudades con grandes ríos, en este aspecto son ciudades privilegiadas. El entorno natural está muy presente en las ciudades de tamaño mediano. La percepción del paisaje cercano tiene una relación con la composición de los espacios urbanos.

Los efectos de asimetría y simetría, de ejes de perspectivas laterales y centrales, de cierre y apertura, convexidad y concavidad, contornos quebrados y ondulados, contrastes y analogías, de puesta en valor de un monumento y anulación de un fondo, de barreras visuales escenografía, y otros, pertenecen tanto al paisaje y al arte urbano como al uso y disfrute de la ciudad, al efecto que las formas producen en el espectador.

Asentamiento Urbano. Por asentamiento urbano se entenderá la radicación de un determinado conglomerado demográfico con el conjunto de sus sistemas de convivencia, en un área físicamente localizada considerando dentro de la misma los elementos naturales y las obras materiales que la integran. Las edificaciones conectadas con la infraestructura y provistas de servicios forman los distintos asentamientos en diferentes escalas, la unidad de vivienda, el conjunto de unidades de vivienda; el caserío, el pueblo, la ciudad, la metrópolis.

Infraestructura Urbana. Es el conjunto de equipamiento técnico que convierte el suelo rural en urbano. Se desarrolla en un complejo de líneas y nudos con sistema reticular, con enlaces, puntos de origen y transformaciones, canales de distribución, puntos de intercambio, y otros. Las principales redes son de dos tipos: aquellas destinadas a la captación y distribución de aguas, gas, 
a la producción y distribución de energía eléctrica; a la canalización y eliminación de aguas residuales, y las destinadas a la comunicación directa entre personas y al transporte de mercancías (calles y sistemas de transportes,) y a la comunicación indirecta entre las personas (teléfono, radio, televisión, telégrafo, y otros).

Estructura Urbana. En nuestro medio, la estructura urbana tendrá una aceptación menos amplia, pero más correcta. Nos referimos a la disposición y relación mutua entre las zonas industriales, de vivienda sociales, de transporte y otras zonas funcionales existentes en cualquier ciudad actual. La planificación de la estructura urbana debe garantizar la combinación racional de las zonas funcionales de un organismo urbano único y una relativa estabilidad de las relaciones entre las principales zonas.

Vialidad Urbana. La vía no es solo un espacio especializado de movimientos (flujos vehiculares y peatonales) sino también un espacio complejo de relación, lugar de intercambio y relación de continuidad de las funciones urbanas: Acera - Ancho de vía - Autopistas - Avenida - Calzada Calle - Estacionamiento - Línea de fábrica - Lindero - Nomenclatura - Pasajes peatonales Peatón - Retiro - Sistema vial - Vía - Vía pública - Vías arteriales - Vías arteriales de enlace básico - Vías de enlace secundario - Vías locales vehiculares -Vías locales peatonales.

Servicios Públicos y Equipamientos Urbanos. Conjunto de instalaciones que permite desarrollar actividades distintas de las básicas de trabajar y residir. Abarcan desde infraestructura técnicas (alumbrado, alcantarillado, red de distribución de agua, teléfonos, y otros), hasta instalaciones en el ámbito de barrio o de ciudad (hospitales, escuelas, cementerios,). En general se realiza con cargo a los presupuestos del sector público.

Mobiliario Urbano. Debe buscar una relación armónica con el espacio urbano y reforzar visualmente su sentido espacial y su carácter. Por eso es necesario proporcionar identidad y seguridad a los usuarios de vías y espacios públicos, buscando hacer agradable su permanencia o recorrido, utilizando un mobiliario adecuado a la función y al espacio.

Los Peatones. El movimiento de peatones es importante tal como el de los vehículos, la gente es muy móvil puede acelerar rápidamente y girar de repente, pero está dispuesta a tomar la ruta más fácil sin considerar su seguridad. Las rutas deben pasar alrededor de la zona de actividad y distribuyendo obstáculos y objetos de interés, se puede hacer una ruta más larga que parezca lo 
más natural. Cuanto más rápidamente se mueva la gente, menos espacio necesita. Las rutas para peatones sobre o debajo de las aceras son un problema de difícil solución. Para impedir que la gente desafíe el tráfico es esencial un flujo más fácil de espacios en los cruces, con rampas suaves y amplios pasos subterráneos.

Suelo Urbano. Es aquel que siendo natural (entorno) en primera instancia el hombre lo transforma en el lugar donde se desarrolla su vida diaria y en donde se satisfacen las necesidades prioritarias. Además, es variable dependiendo de épocas, gustos, leyes o normas, y de otros aspectos que la necesidad misma y exigibilidad de transformación lo requieren. El suelo urbano está relacionado con el hombre, es decir, con su vivienda en la sociedad y tiene características definidas, como ser alto, bajo, estrecho, amplio, fluido, estático, dinámico, claro, oscuro, ambiguo, plano, inclinado, etc. Durante las últimas décadas se ha producido un gran crecimiento de los valores de repercusión del suelo, hasta el punto de que en la actualidad éste presente en torno al $46 \%$ del precio de una vivienda media. Al existir el límite del precio máximo de repercusión del suelo urbano se hace muy necesario afinar en variables urbanísticas clave, tales como la densidad edificatoria y la relación de vacíos y espacios libres por vivienda y en factores económicos como son los costes unitarios de urbanización e infraestructura y los gastos derivados del mantenimiento.

Topografía. Técnica que tiene, de acuerdo a Darquea (1998), como finalidad determinar la forma y dimensiones de un terreno o lugar de la superficie terrestre para representarlo gráficamente con todo detalle. La representación plana de una porción de la superficie terrestre constituye un mapa. Mapa topográfico es el que se da conocer los terrenos con todo su detalle, naturales o artificiales; el nombre de plano se reserva para las representaciones gráficas de superficie de poca extensión. Recibe el nombre de levantamiento el conjunto de operaciones que tiene por objeto de determinar la posición relativa de ciertos puntos en la superficie de la tierra. Los levantamientos topográficos se realizan con instrumentos especiales más o menos precisos.

Morfología de las Manzanas. Si conocer las características de la evolución de una trama se convierte en imprescindible para poder intervenirla, será de gran importancia poder comprender los procesos que llevaron a producir la morfología actual del casco. La lectura de la evolución de una estructura urbana puede ser realizada con mucha nitidez mediante el análisis de las diferentes 
formas y dimensiones que toman las manzanas de un área determinada. En efecto son fácilmente legibles las diferentes etapas de la evolución de un tejido urbano mediante un análisis morfológico, observando sus formas y dimensiones.

Morfología de los Espacios Urbanos. Los espacios públicos de los cascos, explica Marcano (1994), son los elementos que los estructuran, pueden ser comprendidos y una acción de revitalización tendrá que basarse en la compresión de su papel, su morfología al igual a las de las manzanas; es el producto de la manera como se desarrolló el casco, el rol del espacio plaza, su relevancia y su forma de relacionarse con el resto de la estructura. Los otros espacios públicos, calles, plazas secundarias, callejones, pasajes, y otros espacios residuales accidentales, se articulan a la red de espacios del casco formando la malla de los espacios comunitarios. La forma y las dimensiones de los espacios públicos con sus valores de escala y jerarquía juegan un papel de gran importancia. Estas dos variables dependen de sus bordes, los cuales están limitados por las edificaciones. En los cascos las condiciones antes descritas de edificaciones continuas, sin retiros laterales, hace que una acción sobre el espacio público incida directamente sobre el borde construido. Una intervención deberá asegurar las condiciones necesarias para reforzar el casco, uniformidad de la malla edificada, limitaciones de alturas, alineamiento de fachadas, eliminación de los retiros laterales y reforzamiento de los usos mezclados, comercio y oficinas conviviendas.

\section{Metodología}

La investigación fue de tipo descriptivo y documental. Las investigaciones documentales según Arias (2012), es un proceso basado en la búsqueda, recuperación, análisis, crítica e interpretación de datos secundarios, es decir, los obtenidos y registrados por otros investigadores en fuentes documentales: impresas, audiovisuales o electrónicas con el propósito de dar aporte de nuevos conocimientos y es descriptiva por cuanto los hechos se describen tal y como ocurren en la realidad sustentándose en lo planteado por Balestrini (2006), quien afirma: "los estudios descriptivos infieren la descripción acerca de las singularidades de una realidad estudiada"(p.6). Bajo una modalidad de campo, en tanto que la información se obtuvo a través de un estudio sistemático de los hechos en el mismo borde costero del cantón Jaramijó y para ampliar, conocer y profundizar la información se utilizaron libros, revistas y periódicos como medio bibliográfico de información y datos que permitieron generar los resultados expuestos, todos estos 
instrumentos de generación de información constituyeron el corpus del estudio. La técnica para la interpretación y presentación de la información fue la hermenéutica.

\section{Resultados.}

Posterior a la información obtenida fue posible deducir los siguientes resultados:

1. La inexistencia de estudios que consideren los problemas urbanístico y políticos del borde costero del cantón Jaramijó, para de esta forma superar las diferencias sociales de acuerdo a su origen e importancia que habitan en el sector, alejando la posibilidad de un análisis crítico y colectivo que se pueda aplicar y permita una aproximación de su realidad histórica para adaptarla a las necesidades del presente y del futuro.

2. Posterior a la visita del sector se percibe un deterioro general de los servicios básicos y equipamientos urbanos que han causado un proceso de configuraciones morfológicas espacial originadas por cambios cuantitativos y cualitativos en la estructura urbana del borde costero.

3. Las playas del borde costero, están en estado de vulnerabilidad de los riegos naturales, previstos en la no planificación de sus áreas de expansión, al mal uso del suelo y la falta de expresión cultural que valorice la riqueza de su contexto urbanístico, originado un escaso y paulatino crecimiento social-económico y turístico, aunado al desconocimiento de los fines acordados a través de normas y ordenanzas, dando motivo a nuevos protagonismo y estragos en el desarrollo del cantón.

4. Se hizo notorio la falta de políticas claras por parte de las autoridades de turnos y el rol de protagonismo que deben de cumplir dentro de los procesos urbanos del borde costero en detrimento de darle identidad al cantón Jaramijó.

5. Asimismo, cabe señalar que la densidad poblacional del borde costero ha aumentado en un 27\%, lo que exige incrementar servicios para satisfacer necesidades como: servicios básicos, equipamientos urbanos, transportes públicos, vías primarias y secundarias, y espacios públicos que ejerzan modos elementales al uso de sus habitantes. 
6. Con base a la información anteriormente expuesta se propone un plan de transformación para el borde costero de Jaramijó en pro del desarrollo del cantón, en los siguientes términos:

6.1. Criterios y principios de la propuesta del plan: al considerar los aspectos del borde costero para el desarrollo del cantón de Jaramijó se debe prestar atención a los siguientes criterios, según Rueda (1997):

- $\quad$ Plan de densidades, teniendo en cuenta áreas de lotes y manzanas que existen alrededor del área de estudio a fin de organizar una mejor presentación en el espacio urbano y el uso del suelo.

- Definición de la malla vial principal Manta-Jaramijó con un plan de corrección de parámetros que nos permita analizar las posibles ampliaciones de esta importante vía principal, garantizar el acceso permanente y el transporte público y emergente.

- Definición de áreas urbanas y de protección ambiental.

- Definición del espacio público, áreas verdes y recreativas en lo posible con criterios de ubicación equitativa, de accesibilidad y funcionalidad, pensando en la realización de actividades de recreación, deportivas para niños, jóvenes y adultos.

- $\quad$ Plan mejoramiento de las fachadas de las viviendas en función de su ubicación dentro del asentamiento de sus alrededores del área a intervenir y a la malla vial. La introducción de nuevos usos complementarios con la vivienda (pérgolas áreas verdes y otros) dinamiza el asentamiento brindando una presentación a la comunidad y existiendo la posibilidad de obtener una mayor calidad de vida, gracias al intercambio de servicios.

- Plan de servicios públicos utilizando la definición de prioridades en la dotación de servicios e infraestructura básicos junto con la construcción del sistema progresivo de los servicios.

6.2. Lineamientos estratégicos: consolidar la estructura urbana del borde costero como un centro de Servicios para el desarrollo de las actividades productivas, turísticas, recreativas, deportivas, socioculturales del cantón. 
- Lograr el desarrollo urbano de acuerdo con los lineamientos propuestos en este plan, cuidando el entorno natural y paisajístico.

- Estructurar la imagen del borde costero de Jaramijo mediante el Plan de Transformación orientado a la recuperación de la morfología urbana del área costera de la ciudad y por ende al crecimiento y desarrollo del Cantón

6.3. Propuestas de acciones: a falta de políticas compatibles y con las necesidades de ciudades sustentables, se busca generar posibilidades que reflejen las demandas y sus derechos como sociedad local considerando al ser humano como agente de cambio y solventar los problemas que se tienen en relación con los espacios físicos de infraestructura básica y equipamiento de la ciudad.

6.4. Planteamiento estratégico: se debe hacer un planteamiento estratégico, que tenga sustentabilidad y un proceso evolutivo que permita racionalizar y ordenar el uso del suelo del borde costero mediante los planes de equipamientos, servicios básicos, uso del paisaje urbano, vial, recreacional, servicios públicos y turístico, para el mejoramiento de la calidad de vida de sus habitantes y buscar las soluciones en base a una investigación que abarque los siguientes campos.

- $\quad$ Planificación y tratamiento al uso del suelo del borde costero.

- Zonificación que acoja a las diferentes necesidades y actividades del sector.

- Abarcar todos los niveles de equipamiento, servicios, turismo y recreación.

- $\quad$ Articulación del paisaje natural y urbano

- Participación ciudadana.

- Recuperación del patrimonio cultural arquitectónico

- $\quad$ Existencia de seguridad ciudadana.

- $\quad$ Solución inmediata a la insalubridad. 


\section{Conclusiones}

Entre las principales conclusiones se pueden mencionar:

-No existe un ordenamiento, en esta zona de investigación debido a su gran extensión y alta densidad de población esparcida por todas partes, lo cual no ha permitido desarrollar las obras de infraestructuras básicas, los mobiliarios, los equipamientos, la identidad cultural y la falta de un desarrollo urbanístico que le permita consolidarse como una ciudad próspera al desarrollo turístico del cantón.

-El crecimiento del cantón se ha dado en forma espontánea debido a asentamientos ilegales en su periferia, lo cual ha provocado en este sector una imagen deteriorada. La Municipalidad no cuenta con suficiente personal técnico para planificar y controlar el crecimiento de la urbe.

-Ausencia de normas de planificación y de políticas de cuidado del ambiente. Los servicios básicos no cubren la mayoría del territorio y en especial en el área a proponer, estableciendo un caos antes estas necesidades.

-El sistema vial está incompleto, los accesos a la ciudad poseen una trama vial de características irregulares en la parte baja noroccidental, y se torna más regular en su parte sur y sur-oriental. Estas condiciones se acentúan en el eje noroccidental, donde la fluidez y continuidad de las calles se corta debido a la pendiente de la topografía existente. La recolección de basura se realiza dos veces por semana y no existe un tratamiento de los desechos sólidos en el botadero de basura.

-Carencia de espacios verdes, playas descuidadas y escasas áreas de recreación.

-Incipiente tratamiento del espacio urbano público, carencia de mobiliario y equipamiento de la ciudad.

-El sistema eléctrico y de alumbrado público es de pésimas condiciones. La empresa eléctrica provincial (EMELMANABI) cubre toda el área urbana del cantón, pero el problema de mantenimiento de las luminarias se lo realiza desde las oficinas de Manta ya que en Jaramijó solamente existe una oficina de recaudación de valores para el cobro de planillas de luz. 
-El asentamiento poblacional en zonas de la urbe ha provocado una segregación en los barrios debido a las faltas de servicio y del equipamiento de la ciudad, es necesario por esto planificar el desarrollo armónico del cantón.

-El agua potable se distribuye en convenio con la Empresa de Aguas de Manta (EPAM) y es insuficiente para el requerimiento de la población.

-Las redes de alcantarillado cubren únicamente la zona central más poblada.

-La ciudad está servida por una sola línea de buses que recorre la vía Jaramijó - Manta.

-No existen servicios de transporte desde Jaramijó a Montecristi, ni hacia Portoviejo. Los transportes que van por la vía Manta - Jaramijó - Rocafuerte no entran a la zona urbana consolidada.

-Carencia de equipamiento para favorecer servicios turísticos.

-Existen asentamientos irregulares en la zona urbana y periférica, que no tiene legalizada su tenencia de tierra.

\section{Referencias Bibliográficas}

Arias (2012). El proyecto de investigación, Introducción a la metodología científica. Quinta Edición. Editorial Episteme. Caracas

Balestrini (2006). Introducción a la Investigación Pedagógica. México: McGraw - Hill. Interamericana Editores, S.A.

Baruta y el Hatillo. (1994). Revista Urbana. Caracas. pp. 121-134

Darquea, G (1998). Planificación estratégica participativa Municipal. El plan local estratégico participativo: guía metodológica. IULA.

https://books.google.com.ec/books/about/Planeaci\%C3\%B3n_estrat\%C3\%A9gica_participativa_ m.html?id=1U6yAAAAIAAJ\&redir_esc=y

Marcano, F. (1994). Cascos Urbanos: Espacio de reflexión. Los Cascos de Chacao. 
Órgano de difusión del gobierno Municipal del Cantón de Jaramijó (2015). Despacho de la Alcaldía, en la ciudad de Jaramijó.

Romero J (2010). Latinoamérica. Las ciudades y las ideas. Colección Historia y cultura

Historia y cultura. Tercera Edición. Editorial: Siglo Veintiuno Editores. Pag.56 -67

Rueda, S (1997). Metabolismo y Complejidad del Sistema Urbano a la Luz de la Ecología. http:/habitat.aq.upm.es/cs/p2/a008.html. 